\title{
No Significant Changes of Glycemic Control and Renal Function in Patients with Advanced-Stage Diabetic Kidney Disease by Switching from Linagliptin to Teneligliptin
}

\author{
Keiji Hirai (D) \\ Hiroaki Nonaka \\ Moeka Ueda \\ Junki Morino \\ Shohei Kaneko (D) \\ Saori Minato \\ Yuko Mutsuyoshi \\ Katsunori Yanai \\ Hiroki Ishii (iD \\ Momoko Matsuyama \\ Taisuke Kitano \\ Akinori Aomatsu \\ Haruhisa Miyazawa \\ Kiyonori Ito \\ Yuichiro Ueda \\ Susumu Ookawara \\ Yoshiyuki Morishita \\ Division of Nephrology, First \\ Department of Integrated Medicine, \\ Saitama Medical Center, Jichi Medical \\ University, Saitama, Japan
}

\begin{abstract}
Purpose: We compared the efficacy of teneligliptin versus linagliptin for glycemic control and renoprotection in patients with advanced-stage diabetic kidney disease.

Patients and Methods: Changes in the glycated hemoglobin (HbAlc), fasting blood glucose concentration, urine albumin-to-creatinine ratio (UACR), and estimated glomerular filtration rate (eGFR) during a 12-month period were retrospectively analyzed after switching from linagliptin to teneligliptin in 13 patients with advanced-stage diabetic kidney disease (teneligliptin group). Thirteen propensity score-matched patients who were treated with linagliptin alone served as controls (linagliptin group).

Results: The HbA1c, fasting blood glucose concentration, and UACR did not change during the 12-month study period in either group. The annual change rate in the eGFR did not differ between before and after baseline in either group.

Conclusion: Switching from linagliptin to teneligliptin may not improve glycemic control, reduce urinary protein excretion, or ameliorate the rate of renal function decline in patients with advanced-stage diabetic kidney disease. These results suggest that teneligliptin may not be more advantageous for glycemic control and renoprotection compared with linagliptin in patients with advanced-stage diabetic kidney disease.
\end{abstract}

Keywords: chronic kidney disease, diabetic kidney disease, teneligliptin, linagliptin

\section{Introduction}

Diabetic kidney disease is a major complication of type 2 diabetes mellitus and is the leading cause of end-stage renal failure worldwide. ${ }^{1}$ Inadequate blood glucose control is mainly responsible for the development and progression of diabetic kidney disease. ${ }^{2}$ Therefore, optimal glycemic control is necessary to prevent the development and progression of diabetic kidney disease. ${ }^{3}$ However, the use of blood glucose-lowering agents is restricted in patients with advanced diabetic kidney disease because renal dysfunction attenuates the clearance and metabolism of these drugs and increases the risk of hypoglycemia. ${ }^{4}$

A dipeptidyl peptidase-4 (DPP-4) inhibitor is an oral hypoglycemic drug that enhances insulin secretion from pancreatic beta cells and inhibits glucagon secretion from alpha cells depending on the blood glucose concentration. ${ }^{5}$ Therefore, DPP-4 inhibitors can be safely used in patients with renal impairment. Among DPP-4
Correspondence: Keiji Hirai

Division of Nephrology, First Department of Integrated Medicine, Saitama Medical Center, Jichi Medical University, Saitama, Japan

Tel +8I-48-647-2 III

Fax +8I-48-647-683I

Email keijihirai@kfy.biglobe.ne.jp 
inhibitors, linagliptin and teneligliptin do not need dose adjustment in patients with renal impairment; ${ }^{6}$ therefore, these two drugs can be safely and easily used in patients with advanced-stage diabetic kidney disease. Teneligliptin reportedly has effects comparable with those of linagliptin on glycemic control in patients with concurrent type 2 diabetes and renal impairment. ${ }^{7}$ Linagliptin has been shown to reduce urinary albumin excretion in these patients. ${ }^{8}$ However, few studies have been performed to assess the renoprotective effects of teneligliptin in patients with concurrent type 2 diabetes and renal impairment. Therefore, in this study, we compared the efficacy of teneligliptin versus linagliptin for glycemic control and renoprotection in patients with advanced-stage diabetic kidney disease.

\section{Materials and Methods}

\section{Ethical Approval}

This study was approved by the ethics committee of Saitama Medical Center, Jichi Medical University (RIN 15-33) and was conducted according to the ethical principles contained within the Declaration of Helsinki. Informed consent was not applicable because of the retrospective nature of the study. Information regarding this study was displayed on notice boards in the patient waiting rooms of our institution to provide all patients with their right to opt out.

\section{Patients}

We retrospectively analyzed the data of patients who had been treated at Saitama Medical Center, Jichi Medical University from 2015 to 2019. The inclusion criteria were (i) an age of $\geq 20$ years, (ii) a diagnosis of type 2 diabetic kidney disease with an estimated glomerular filtration rate (eGFR) of $\leq 60 \mathrm{~mL} / \mathrm{min} / 1.73 \mathrm{~m}^{2}$ and a urine albumin-tocreatinine ratio (UACR) of $\geq 0.3 \mathrm{~g} / \mathrm{g} \mathrm{Cr}$, and (iii) a history of having taken linagliptin for at least 24 months or teneligliptin at least 12 months after taking linagliptin for at least 12 months. The exclusion criteria were (i) a diagnosis of type 1 diabetes mellitus or secondary diabetes mellitus; (ii) malignancy, severe infection, or steroid therapy; and (iii) renal replacement therapies including hemodialysis, peritoneal dialysis, and renal transplantation.

\section{Study Design}

This was a retrospective comparative study that was compliant with the Strengthening the Reporting of Observational Studies in Epidemiology statement (Supplemental Table 1). ${ }^{9}$ The study design is illustrated in Figure 1. The patients' demographic and clinical data were retrospectively obtained from their medical records. The teneligliptin group comprised the patients who had been taking teneligliptin at least 12 months after linagliptin for at least 12 months, and the linagliptin group comprised the patients matched for baseline characteristics who had been taking linagliptin for at least 24 months. The baselines of each patient in the linagliptin group were defined on the days from 1 April 2017 to 1 July 2017, during which time linagliptin was changed to teneligliptin in the teneligliptin group. Linagliptin was orally administered at a dosage of $5 \mathrm{mg}$ once daily after breakfast. Teneligliptin was orally administered at a dosage of $20 \mathrm{mg}$ once daily after breakfast and was up-titrated to $40 \mathrm{mg}$ if glycated hemoglobin $(\mathrm{HbA} 1 \mathrm{c})$ did not improve after the initiation

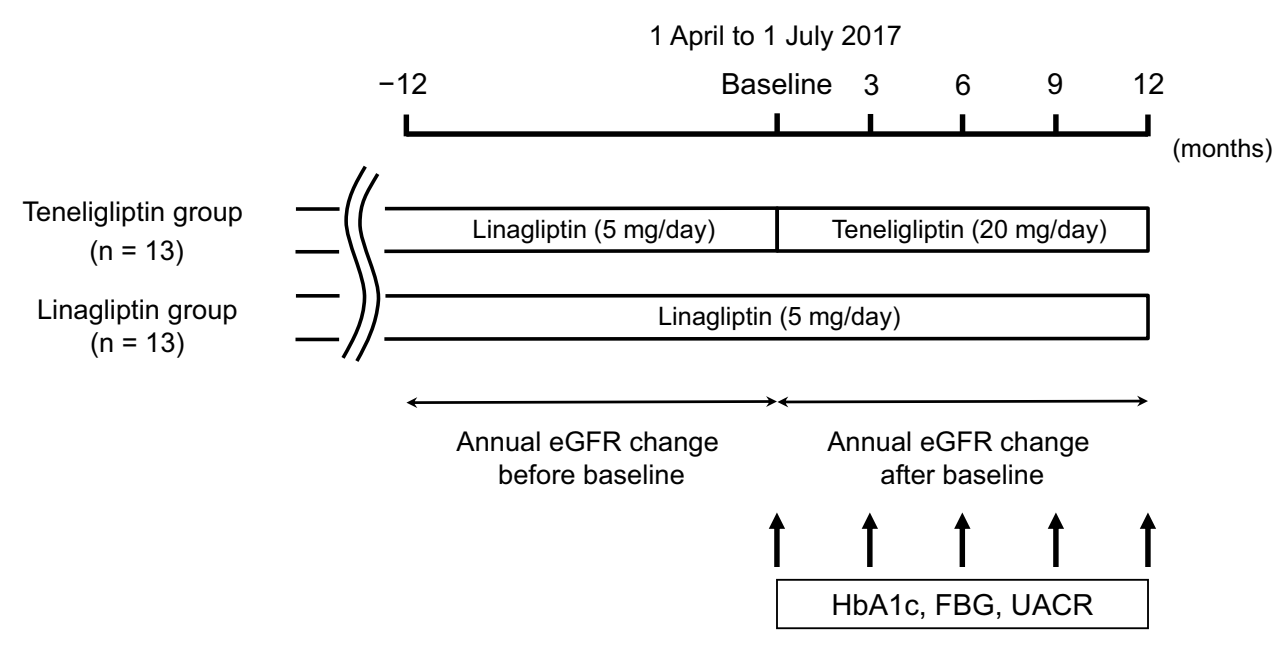

Figure I Study design.

Abbreviations: eGFR, estimated glomerular filtration rate; FBG, fasting blood glucose; HbAlc, glycated hemoglobin; UACR, urine albumin-to-creatinine ratio. 
of teneligliptin. This study was performed in a clinical setting, and all tests and treatments were covered under medical insurance. Drugs including linagliptin and teneligliptin were not purchased from any companies nor were provided by them. Changes in the HbAlc concentration, fasting blood glucose, and UACR at 3, 6, 9, and 12 months were compared with those at baseline in each group and were compared between the two groups. The annual changes in the eGFR were evaluated 12 months before and after the baseline in each group.

\section{Laboratory Methods}

Blood and urinary parameters were measured by the Department of Clinical Laboratory, Saitama Medical Center. The eGFR was calculated by the Modification of Diet in Renal Disease study equation modified for Japanese patients with chronic kidney disease as follows: eGFR $\left(\mathrm{mL} / \mathrm{min} / 1.73 \mathrm{~m}^{2}\right)=194 \times$ age $-0.287 \times$ serum creatinine -1.094 (multiplied by 0.739 for female).$^{10}$ The annual change rate in the eGFR was determined using linear regression analysis as the slope per month for each patient before and after baseline.

\section{Statistics}

Statistical analyses were performed using JMP v11 (SAS Institute, Cary, North Carolina, USA). Propensity score matching analysis was used to select control patients with similar baseline characteristics. Age, sex, body mass index, $\mathrm{HbAlc}$ concentration, eGFR, and UACR were included in the propensity model as independent variables. One-to-one pair matching was performed by identifying a control patient with the nearest log-transformed propensity score with a caliper width of 0.25 standard deviation. The resulting score-matched pairs were used in subsequent analyses. Data were expressed as mean \pm standard deviation for continuous variables and as number and percentage for categorical variables. Clinical parameters were compared between two groups using Student's $t$-test. Component ratios were compared between two groups using Fisher's exact test. Serial measurements were compared within each group using repeated-measures analysis of variance with Tukey's test. A P value of $<0.05$ was considered statistically significant. The post hoc power analysis was performed to assess the statistical power to detect a difference between the two groups. Power calculations were based on a two sample $t$-test for paired data using a two-sided significance level of 0.05 .

\section{Results}

\section{Patient Characteristics}

In total, 233 patients with type 2 diabetes who had an eGFR of $\leq 60 \mathrm{~mL} / \mathrm{min} / 1.73 \mathrm{~m}^{2}$ and UACR of $\geq 0.3 \mathrm{~g} / \mathrm{g} \mathrm{Cr}$ were identified. Twenty-eight of these patients were taking teneligliptin, and 32 were taking linagliptin. Among the patients taking teneligliptin, 6 did not meet the inclusion criteria and 3 met the exclusion criteria. Eight of the patients taking linagliptin met the exclusion criteria. After propensity score matching, 13 pairs of patients were included (Figure 2) (Supplemental Table 2). In total, 26 patients were analyzed (14 men and 12 women; mean age, $71.7 \pm$ 10.3 years). Their mean eGFR at baseline was $24.2 \pm$ $9.1 \mathrm{~mL} / \mathrm{min} / 1.73 \mathrm{~m}^{2}$, and their chronic kidney disease stages were as follows: stage G3b in 7 (26.9\%) patients, stage G4 in $13(50.0 \%)$, and stage G5 in $6(23.1 \%)$. Their mean $\mathrm{HbAlc}$ concentration and UACR at baseline were $6.9 \pm$ $0.6 \%$ and $1.3 \pm 1.4 \mathrm{~g} / \mathrm{g} \mathrm{Cr}$, respectively. Finally, 13 patients were categorized into the teneligliptin group and 13 patients were categorized into the linagliptin group. The patients' baseline characteristics and medications in the two groups are summarized in Table 1. There was no significant difference in any clinical parameters between the two groups. The administered dosage of teneligliptin was $20 \mathrm{mg}$ /day in 11 patients and $40 \mathrm{mg} /$ day in 2 patients. The administered dosage of linagliptin was $5 \mathrm{mg} /$ day in 13 patients.

\section{Effect of Teneligliptin on Glycemic Control}

The HbAlc concentration at 3, 6, 9, and 12 months was not different from that at baseline in both the teneligliptin and linagliptin groups (Figure 3). Fasting blood glucose concentration at 3, 6, 9, and 12 months did not change from baseline in both the teneligliptin and linagliptin groups (Figure 4). The mean difference in $\mathrm{HbAlc}$ concentration at 12 months was $0.2 \%$, and a standard deviation was $0.5 \%$. Post hoc power analysis showed $17 \%$ statistical power to detect a difference in $\mathrm{HbAlc}$ concentration.

\section{Effects of Teneligliptin on UACR and Annual Change Rate in eGFR}

The UACR at 3, 6, 9, and 12 months remained unchanged from baseline in both the teneligliptin and linagliptin groups (Figure 5). The mean difference in UACR at 12 months was $0.1 \mathrm{~g} / \mathrm{g} \mathrm{Cr}$, and a standard deviation was $0.9 \mathrm{~g} /$ $\mathrm{g}$ Cr. Post hoc power analysis showed 5\% statistical power to detect a difference in UACR. The annual change rate in 


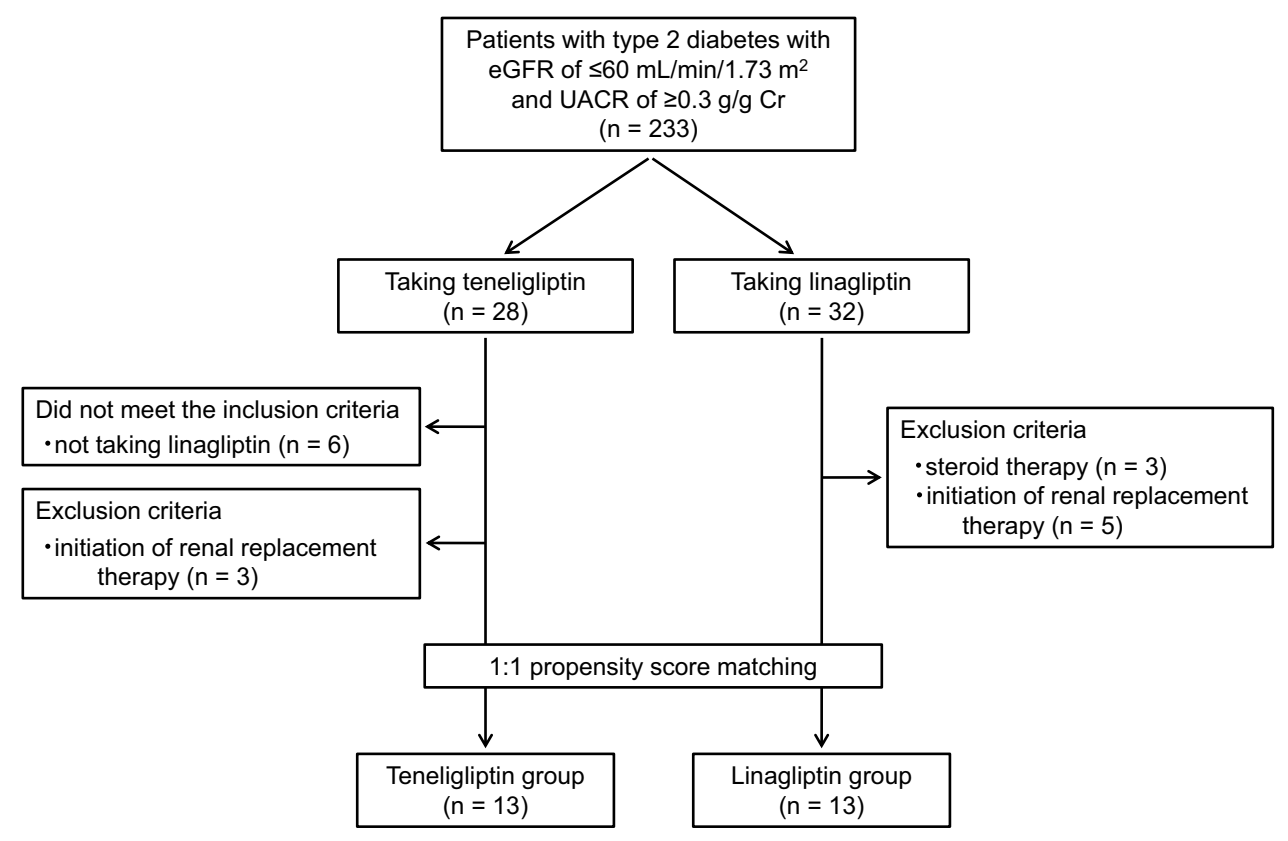

Figure 2 Patient flow diagram.

Abbreviations: eGFR, estimated glomerular filtration rate; UACR, urine albumin-to-creatinine ratio.

the eGFR did not differ between before and after baseline in both the teneligliptin and linagliptin groups (Figures 6 and 7). The annual change rate in the eGFR after baseline was not different between the two groups. The mean difference in annual change rate in eGFR after baseline was $0.4 \mathrm{~mL} / \mathrm{min} / 1.73 \mathrm{~m}^{2} /$ year, and the standard deviation was $5.0 \mathrm{~mL} / \mathrm{min} / 1.73 \mathrm{~m}^{2} /$ year. Post hoc power analysis showed $4 \%$ statistical power to detect a difference in annual change rate in eGFR.

\section{Changes in Other Clinical Parameters}

Other clinical and laboratory parameters, including body weight, systolic blood pressure, low-density lipoprotein cholesterol, high-density lipoprotein cholesterol, triglyceride, uric acid, albumin, hemoglobin, sodium, potassium, chloride, calcium, and phosphate, were not significantly different at 3, 6, 9, and 12 months versus baseline in either the teneligliptin or linagliptin group (data not shown).

\section{Discussion}

In the present study, we compared the efficacy of teneligliptin versus linagliptin for glycemic control and renoprotection in patients with advanced-stage diabetic kidney disease. We found that switching from linagliptin to teneligliptin did not improve glycemic control, reduce urinary protein excretion, or ameliorate the rate of renal function decline. These results suggest that teneligliptin and linagliptin are similarly effective for glycemic control in patients with advanced-stage diabetic kidney disease.

Teneligliptin is a potent, selective, and long-acting DPP-4 inhibitor that is available in Japan for the treatment of type 2 diabetes. ${ }^{6}$ Teneligliptin is metabolized in the liver and excreted via dual hepatic and renal routes; therefore, it needs no dose adjustment for use in patients with renal impairment. ${ }^{6}$ A double-blind randomized clinical study showed that teneligliptin significantly reduced the HbA1c concentration compared with placebo in patients with type 2 diabetes mellitus and normal renal function. ${ }^{11}$ Teneligliptin is reportedly as effective as linagliptin for glycemic control in patients with concurrent type 2 diabetes and renal impairment. ${ }^{7}$ In the present study, the HbA1c concentration did not change after switching from linagliptin to teneligliptin. Teneligliptin has been shown to reduce the blood glucose concentration in a dose-dependent manner, ${ }^{11}$ and it can be increased to a high dosage (40 $\mathrm{mg}$ once daily) if the standard dosage (20 mg once daily) is insufficient for glycemic control. ${ }^{6}$ In our study, $85 \%$ of patients were treated at a dosage of $20 \mathrm{mg} /$ day, and only $15 \%$ of patients were treated at a dosage of $40 \mathrm{mg} /$ day. The use of teneligliptin at a submaximal dose in our study might explain why teneligliptin did not reduce the HbAlc concentration after changing from linagliptin. Further large-scale prospective randomized controlled studies are necessary to clarify the 
Table I Baseline Patient Characteristics

\begin{tabular}{|c|c|c|c|}
\hline & Teneligliptin Group $(n=13)$ & Linagliptin Group $(n=13)$ & $P$ value \\
\hline Age (years) & $74.8 \pm 8.3$ & $68.6 \pm 11.5$ & 0.13 \\
\hline Sex (male/female) & $7 / 6$ & $7 / 6$ & 1.00 \\
\hline Body mass index $\left(\mathrm{kg} / \mathrm{m}^{2}\right)$ & $25.2 \pm 3.3$ & $24.3 \pm 3.0$ & 0.44 \\
\hline Systolic blood pressure $(\mathrm{mmHg})$ & $138.3 \pm 9.1$ & $135.5 \pm 19.3$ & 0.64 \\
\hline Diastolic blood pressure $(\mathrm{mmHg})$ & $73.9 \pm 11.2$ & $70.4 \pm 12.0$ & 0.44 \\
\hline Serum creatinine $(\mathrm{mg} / \mathrm{dL})$ & $2.4 \pm 1.0$ & $2.1 \pm 1.0$ & 0.42 \\
\hline eGFR $\left(\mathrm{mL} / \mathrm{min} / 1.73 \mathrm{~m}^{2}\right)$ & $22.6 \pm 9.4$ & $25.8 \pm 8.9$ & 0.47 \\
\hline \multicolumn{4}{|l|}{ CKD stage (number, \%) } \\
\hline G3b & $3(23.1)$ & $4(30.8)$ & 0.75 \\
\hline G4 & $7(53.8)$ & $6(46.2)$ & \\
\hline G5 & $3(23.1)$ & $3(23.1)$ & \\
\hline Urinary protein excretion $(\mathrm{g} / \mathrm{gCr})$ & $1.0 \pm 1.1$ & $1.7 \pm 1.6$ & 0.32 \\
\hline \multicolumn{4}{|l|}{ History of underlying causes of CKD (number, \%) } \\
\hline Diabetic nephropathy & $8(61.5)$ & $9(69.2)$ & 0.72 \\
\hline Hypertensive nephrosclerosis & $2(15.4)$ & $3(23.1)$ & \\
\hline Chronic glomerulonephritis & $3(23.1)$ & I (7.7) & \\
\hline Duration of diabetes mellitus (year) & $17.5 \pm 12.8$ & $24.3 \pm 11.9$ & 0.18 \\
\hline HbAlc (\%) & $6.8 \pm 0.7$ & $7.1 \pm 0.6$ & 0.26 \\
\hline Fasting blood glucose (mg/dL) & $132.6 \pm 28.8$ & $146.4 \pm 31.0$ & 0.30 \\
\hline LDL-cholesterol (mg/dL) & $88.5 \pm 21.7$ & $96.4 \pm 28.7$ & 0.44 \\
\hline HDL-cholesterol (mg/dL) & $44.9 \pm 15.2$ & $41.9 \pm 15.5$ & 0.62 \\
\hline Triglyceride $(\mathrm{mg} / \mathrm{dL})$ & $194.6 \pm 113.1$ & $145.2 \pm 83.5$ & 0.22 \\
\hline Uric acid (mg/dL) & $5.8 \pm 1.3$ & $5.8 \pm 1.4$ & 0.95 \\
\hline Albumin $(g / d L)$ & $4.0 \pm 0.4$ & $3.9 \pm 0.4$ & 0.69 \\
\hline Hemoglobin $(g / d L)$ & $11.8 \pm 1.9$ & $11.6 \pm 1.9$ & 0.85 \\
\hline Erythropoiesis-stimulating agent (number, \%) & $3(23.1)$ & $4(30.8)$ & 1.00 \\
\hline Glinide (number, \%) & $3(23.1)$ & $5(38.5)$ & 0.67 \\
\hline$\alpha$-glucosidase inhibitor (number, \%) & I (7.7) & I (7.7) & 1.00 \\
\hline SGLT2 inhibitor (number, \%) & I (7.7) & $2(15.4)$ & 1.00 \\
\hline Insulin (number, \%) & $3(23.1)$ & $4(30.8)$ & 1.00 \\
\hline RAS blocker (number, \%) & $9(69.2)$ & $10(76.9)$ & 1.00 \\
\hline Diuretic (number, \%) & $7(53.8)$ & $4(30.8)$ & 0.43 \\
\hline Statin (number, \%) & $9(69.2)$ & $6(46.2)$ & 0.43 \\
\hline Eicosapentaenoic acid (number, \%) & $3(23.1)$ & $3(23.1)$ & 1.00 \\
\hline Antiplatelet agent (number, \%) & $6(46.2)$ & $8(61.5)$ & 0.70 \\
\hline Antihyperuricemic drug (number, \%) & II (84.6) & $9(69.2)$ & 0.64 \\
\hline
\end{tabular}

Note: Data are presented as mean \pm standard deviation, number, or number (\%).

Abbreviations: CKD, chronic kidney disease; eGFR, estimated glomerular filtration rate; HbAIc, glycated hemoglobin; HDL, high-density lipoprotein; LDL, low-density lipoprotein; RAS, renin-angiotensin system; SGLT-2, sodium-glucose cotransporter-2. 


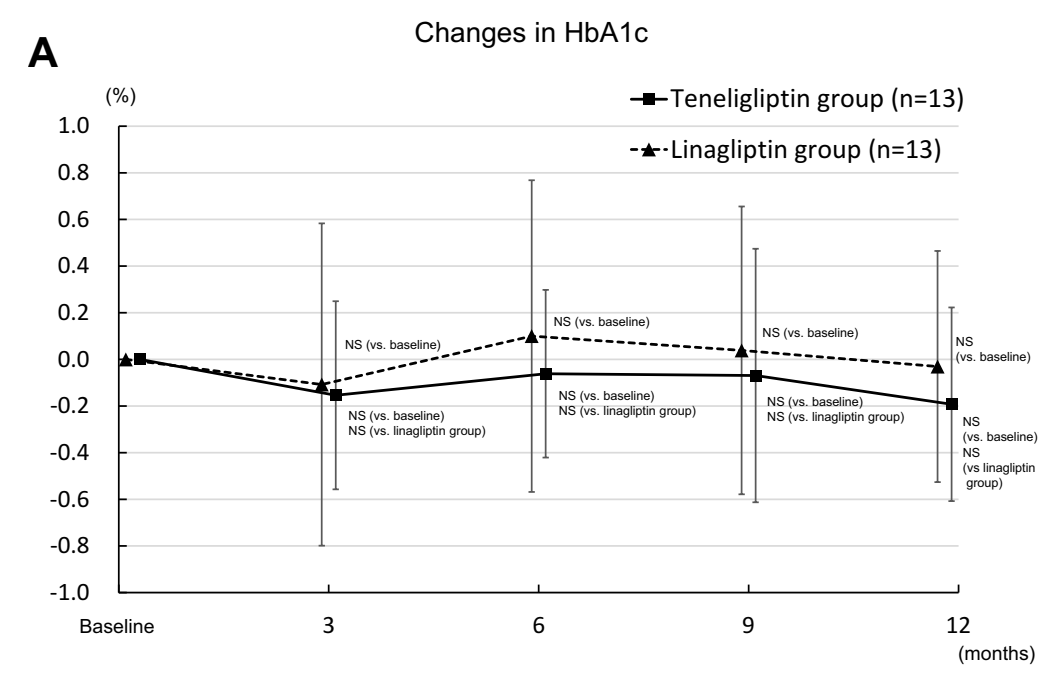

B Changes in $\mathrm{HbA} 1 \mathrm{c}$ in teneligliptin group

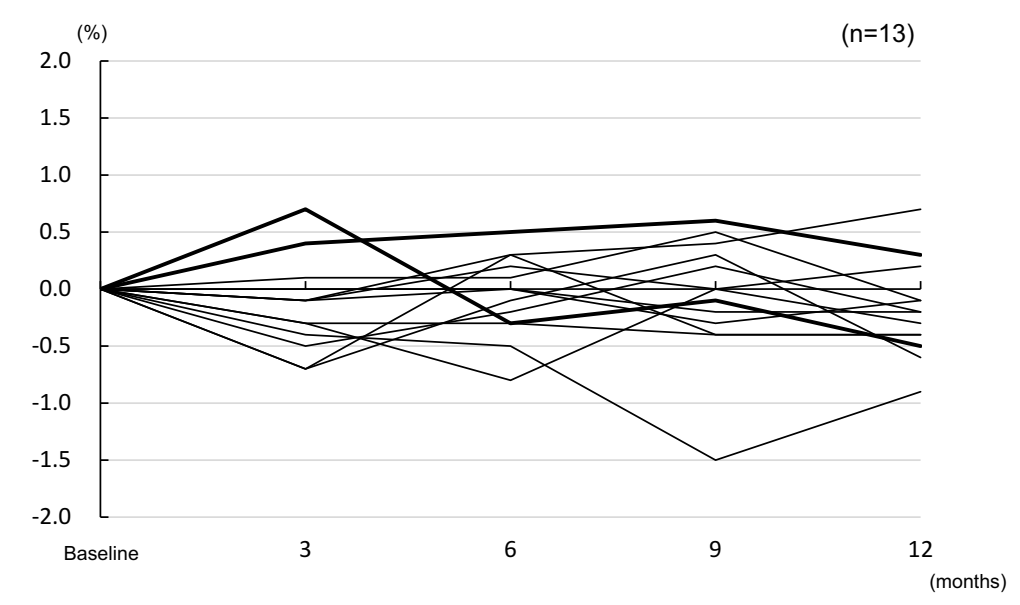

C Changes in $\mathrm{HbA1c}$ in linagliptin group

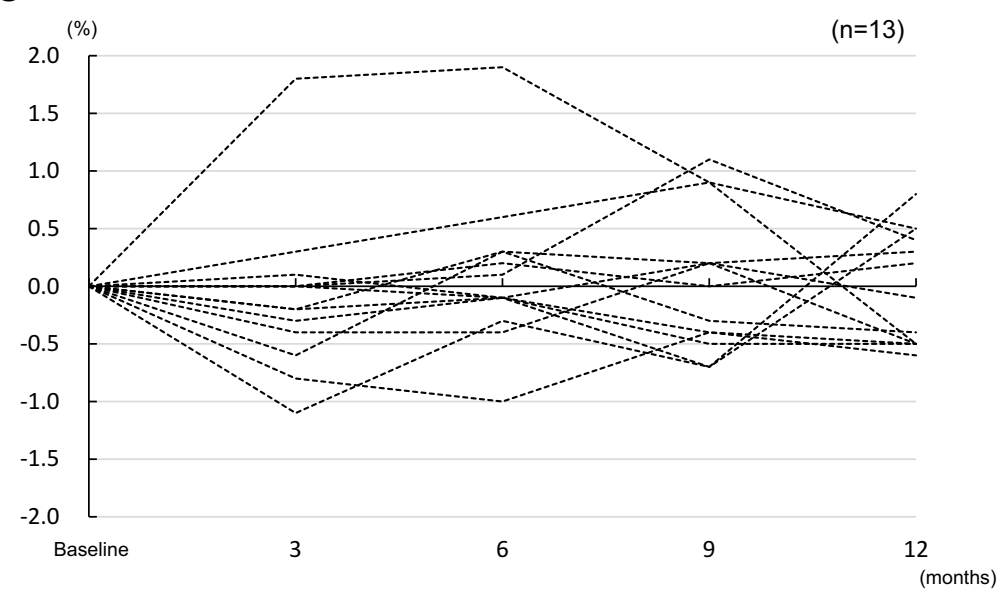

Figure 3 Changes in HbAlc over 12 months following the baseline measurement. (A) Changes in HbAlc in the teneligliptin and linagliptin groups. (B) Changes in HbAlc in the teneligliptin group. Eleven patients were taking $20 \mathrm{mg} /$ day of teneligliptin (thin solid line) and two patients were $40 \mathrm{mg} /$ day (bold solid line). (C) Changes in HbAlc in the linagliptin group.

Abbreviations: HbAIc, glycated hemoglobin; NS, not significant. 

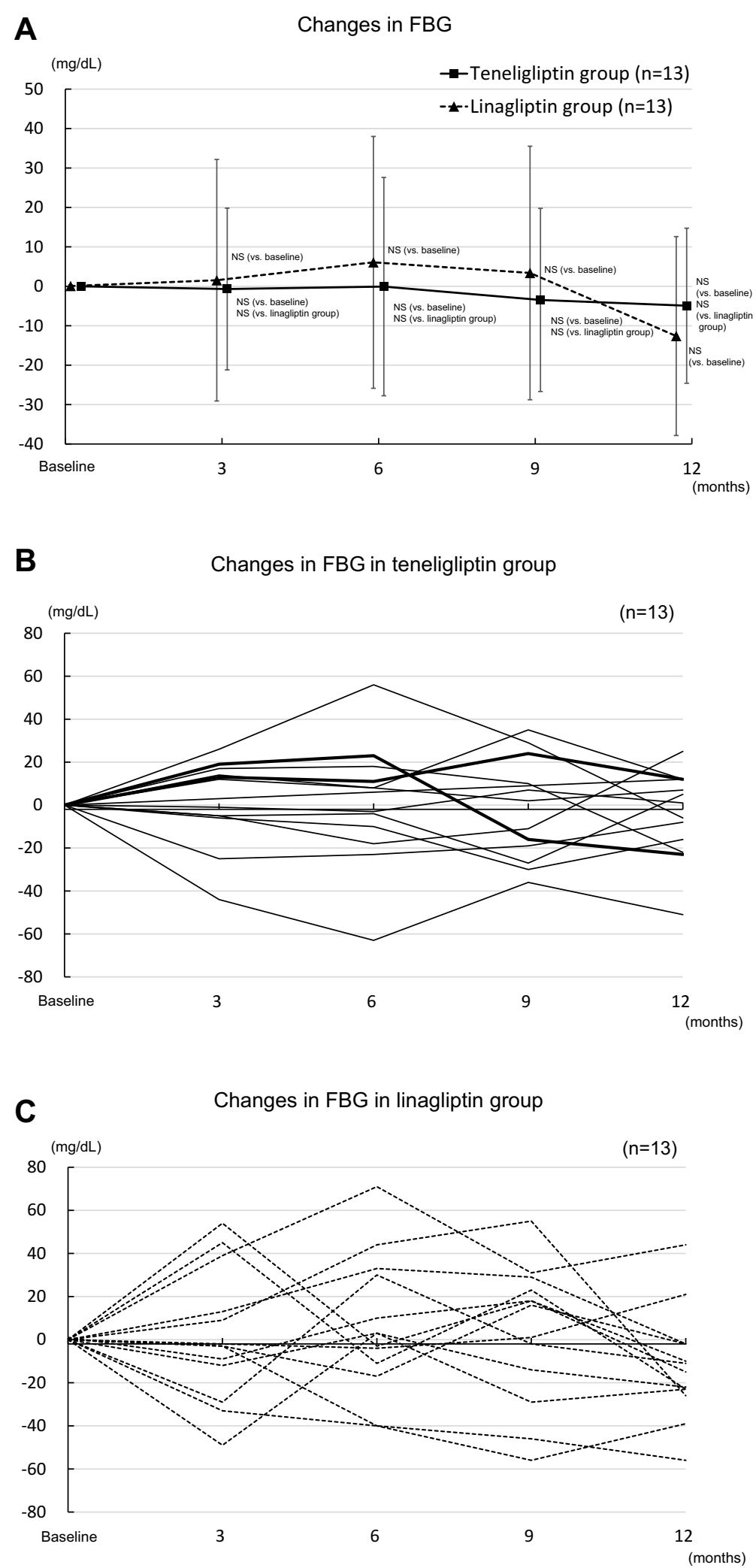

Figure 4 Changes in FBG over 12 months following the baseline measurement. (A) Changes in FBG in the teneligliptin and linagliptin groups. (B) Changes in FBG in the teneligliptin group. Eleven patients were taking $20 \mathrm{mg} /$ day of teneligliptin (thin solid line) and two patients were $40 \mathrm{mg} /$ day (bold solid line). (C) Changes in FBG in the linagliptin group.

Abbreviations: FBG, fasting blood glucose; NS, not significant. 


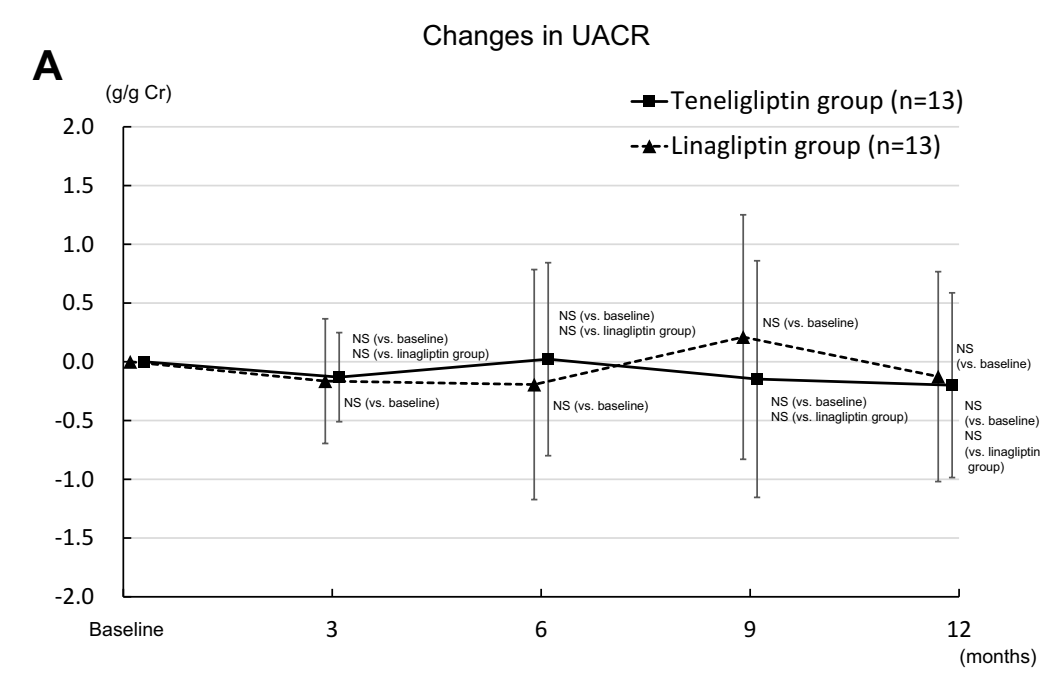

B Changes in UACR in teneligliptin group

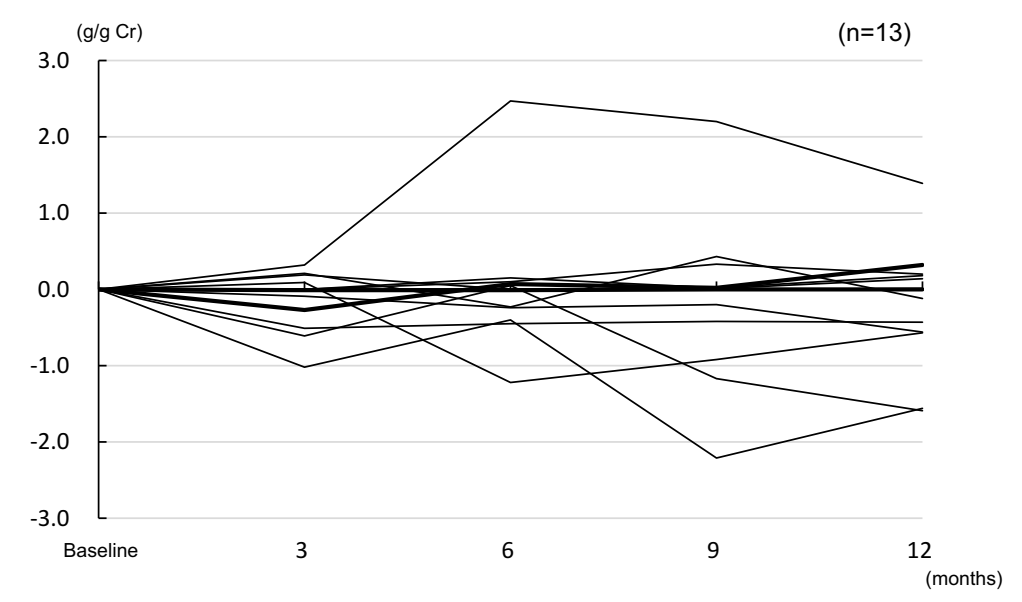

C Changes in UACR in linagliptin group

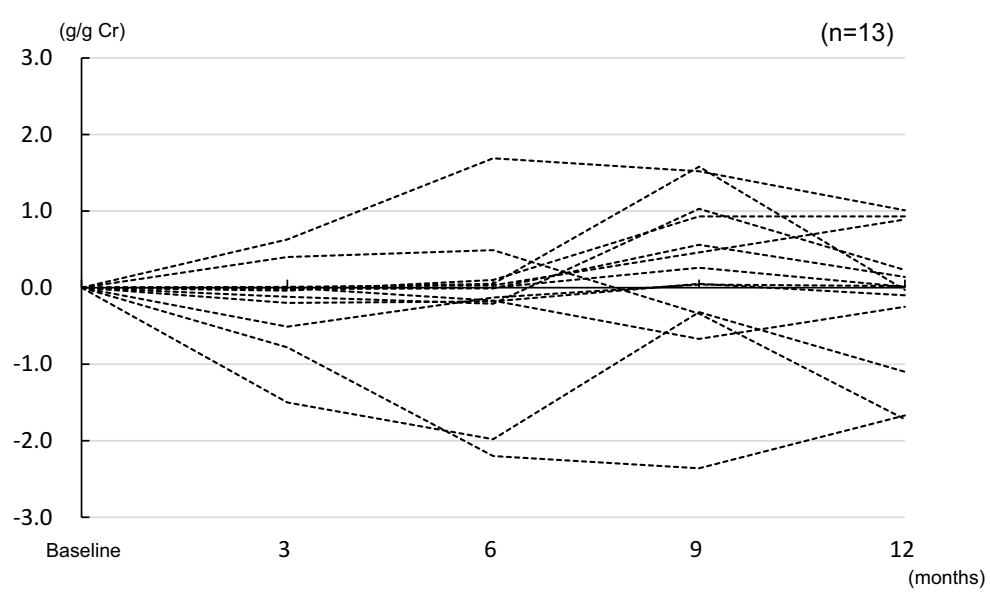

Figure 5 Changes in UACR over 12 months following the baseline measurement. (A) Changes in UACR in the teneligliptin and linagliptin groups. (B) Changes in UACR in the teneligliptin group. Eleven patients were taking $20 \mathrm{mg} /$ day of teneligliptin (thin solid line) and two patients were $40 \mathrm{mg} / \mathrm{day}$ (bold solid line). (C) Changes in UACR in the linagliptin group.

Abbreviations: NS, not significant; UACR, urine albumin-to-creatinine ratio. 


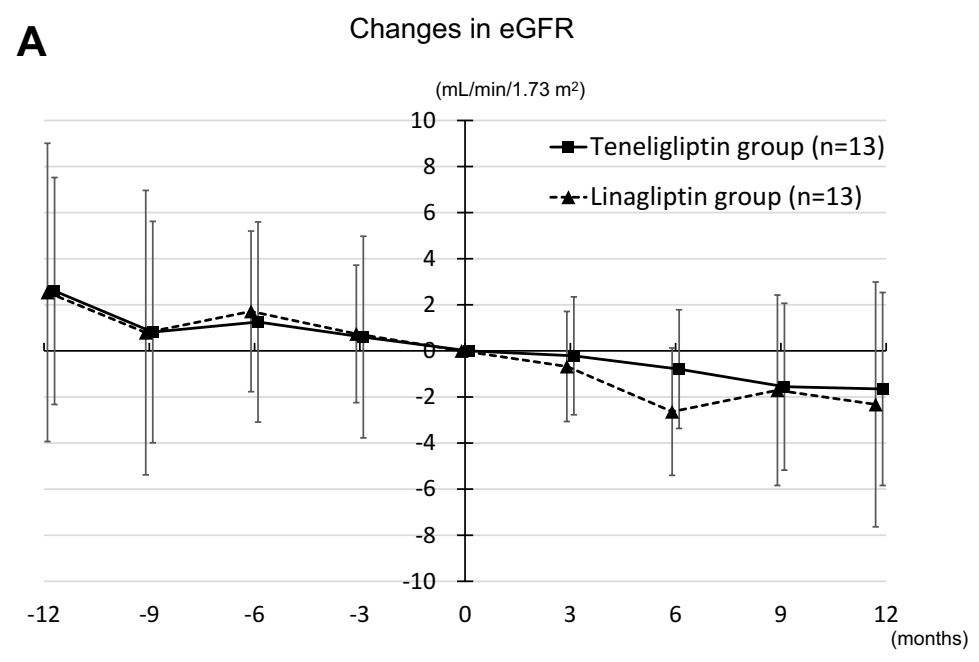

B

Changes in eGFR in teneligliptin group

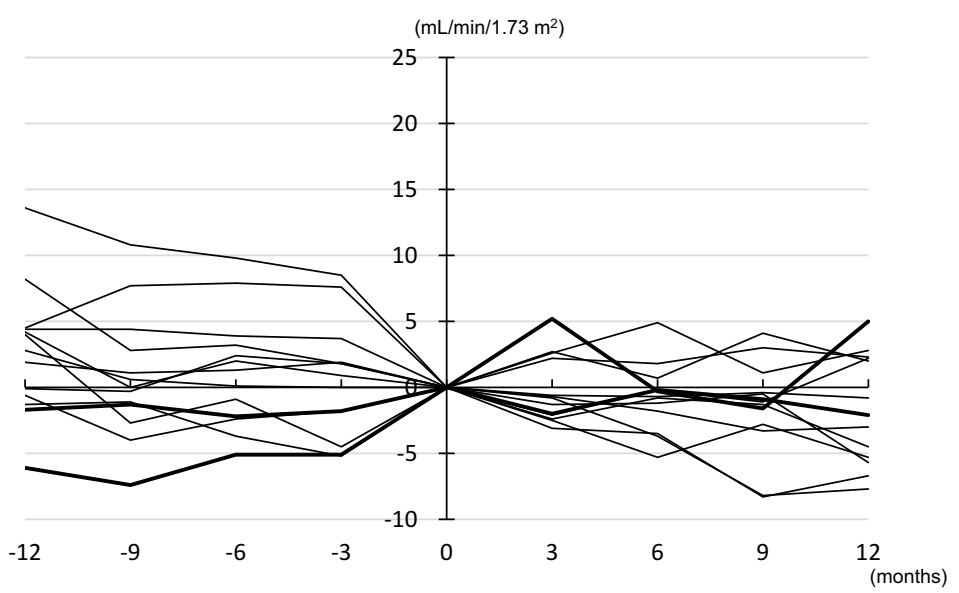

C

Changes in eGFR in linagliptin group

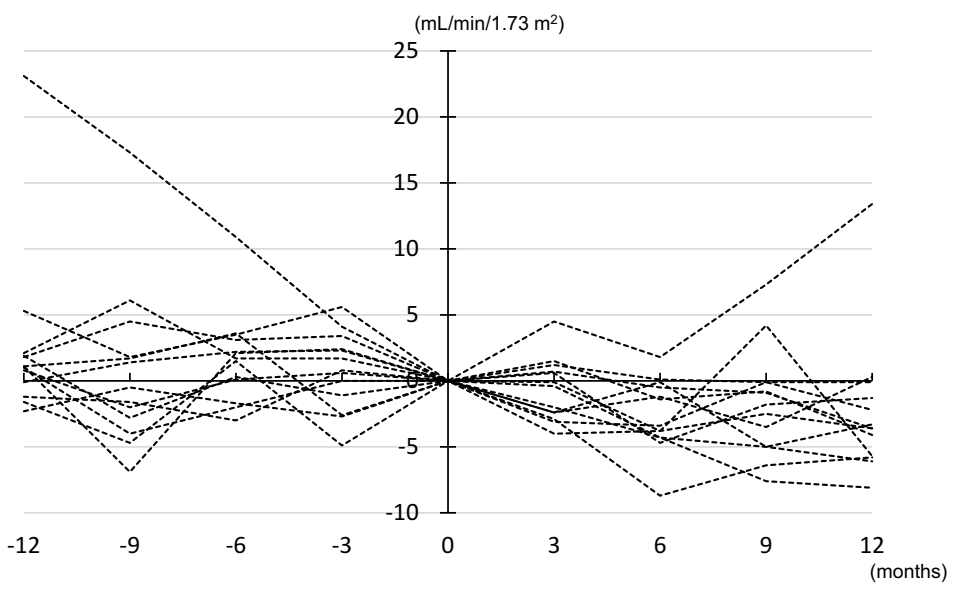

Figure 6 Changes in eGFR over 12 months before and after the baseline measurement. (A) Changes in eGFR in the teneligliptin and linagliptin groups. (B) Changes in eGFR in the teneligliptin group. Eleven patients were taking $20 \mathrm{mg} /$ day of teneligliptin (thin solid line) and two patients were $40 \mathrm{mg} / \mathrm{day}$ (bold solid line). (C) Changes in eGFR in the linagliptin group.

Abbreviation: eGFR, estimated glomerular filtration rate. 


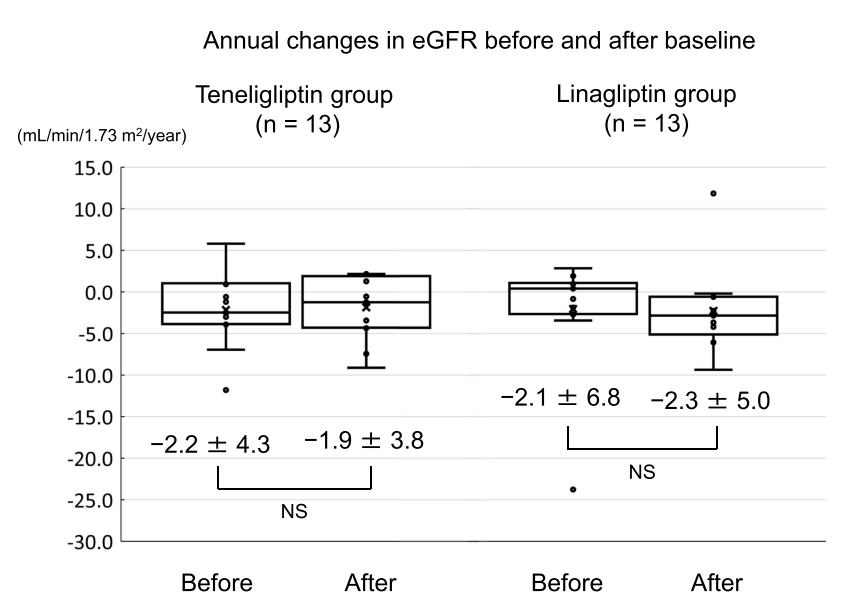

Figure 7 Annual changes in eGFR before and after baseline in the teneligliptin and linagliptin groups.

Abbreviations: eGFR, estimated glomerular filtration rate; NS, not significant.

blood glucose-lowering effect of teneligliptin in patients with advanced-stage diabetic kidney disease.

Several randomized clinical studies have shown that linagliptin reduces urinary protein excretion in patients with concurrent type 2 diabetes and renal impairment. ${ }^{8,12}$ In the present study, urinary protein excretion did not change after switching from linagliptin to teneligliptin. These results suggest that teneligliptin might have a similar effect on proteinuria in patients with advancedstage diabetic kidney disease. Further studies are needed to investigate the anti-proteinuric effect of teneligliptin in patients with advanced-stage diabetic kidney disease.

A recent randomized clinical study showed that linagliptin did not ameliorate the rate of decline in the eGFR in patients with concurrent type 2 diabetes and renal impairment. ${ }^{8}$ In the present study, the annual rate of decline in the eGFR did not change after switching from linagliptin to teneligliptin. These results suggest that teneligliptin might not have a beneficial effect on renal function decline in patients with advanced-stage diabetic kidney disease. Further studies are required to investigate the renoprotective effect of teneligliptin in patients with advanced-stage diabetic kidney disease. In the present study, improvement of glucose metabolism and renal parameters was observed in a part of the patients after switching from linagliptin to teneligliptin. Some patients might respond favorably to teneligliptin treatment. However, the effects of high dose of teneligliptin were unclear in our study. Further studies are required to investigate which patients are likely to benefit from teneligliptin and whether high dose of teneligliptin is beneficial in patients with advanced-stage diabetic kidney disease.
This study had several limitations. First, it was a retrospective observational study; therefore, patient selection bias cannot be completely removed from the study, although propensity score matching was used to control selection bias and balance covariates. Second, the study was performed at a single center, which limits the external validity of the results. Third, the number of participants in the study was small, which may have reduced the statistical power to detect group differences. Therefore, no statistical significance might be due to the under power of our study. Fourth, the reason for choosing each treatment regimen for each patient was different. Also, not all patients were treated with the same dose of teneligliptin. This might cause a significant bias and limit generalizability. Fifth, other hypoglycemic agents, including glinide, $\alpha$-glucosidase inhibitor, sodium-glucose cotransporter-2 inhibitor, and insulin, are concomitantly used in the study. The possibility cannot be excluded that the dosage of these drugs might have been changed during the study period because physicians might have adjusted hypoglycemic medications when they observed better or worse glycemic control. The adverse effects associated with teneligliptin in this study cannot also be assessed because of the retrospective nature of study design. Therefore, multicenter, large-scale, prospective, randomized clinical studies with adequate statistical power are required to clarify the renoprotective efficacy and safety of teneligliptin in patients with advanced-stage diabetic kidney disease.

In conclusion, teneligliptin may not improve glycemic control or show any additional renoprotective effects after switching from linagliptin.

\section{Acknowledgments}

We thank all the outpatient medical staff of our institution for their excellent work. We also thank Angela Morben, DVM, ELS, from Edanz Group, for editing a draft of this manuscript.

\section{Author Contributions}

AA and HM conceived and designed the study. HN, MU, JM, SK, SM, YM (Yuko Mutsuyoshi), MM, and TK collected the data. HI and KY performed the statistical analysis. KH wrote the first draft of the manuscript. KI, YU, and SO made critical revisions. YM (Yoshiyuki Morishita) approved the final version. All authors contributed to this manuscript and approved the final version for submission. All authors contributed to data analysis, drafting or revising the article, have agreed on the journal to which the article will be submitted, gave final approval of 
the version to be published, and agree to be accountable for all aspects of the work.

\section{Funding}

This study was not supported by any funding source.

\section{Disclosure}

The authors report no conflicts of interest in this work.

\section{References}

1. Ghaderian SB, Hayati F, Shayanpour S, Beladi Mousavi SS. Diabetes and end-stage renal disease; a review article on new concepts. $J$ Renal Inj Prev. 2015;4(2):28-33.

2. Tziomalos K, Athyros VG. Diabetic nephropathy: new risk factors and improvements in diagnosis. Rev Diabet Stud. 2015;12(1-2):110-118. doi:10.1900/RDS.2015.12.110

3. Wong MG, Perkovic V, Chalmers J, et al. Long-term benefits of intensive glucose control for preventing end-stage kidney disease: Advance-on. Diabetes Care. 2016;39(5):694-700. doi:10.2337/dc152322

4. Betonico CC, Titan SM, Correa-Giannella ML, Nery M, Queiroz M. Management of diabetes mellitus in individuals with chronic kidney disease: therapeutic perspectives and glycemic control. Clinics (Sao Paulo). 2016;71(1):47-53. doi:10.6061/clinics/2016(01)08

5. Scheen AJ. A review of gliptins for 2014. Expert Opin Pharmacother. 2015;16(1):43-62. doi:10.1517/14656566.2015.978289
6. Ceriello A, De Nigris V, Iijima H, Matsui T, Gouda M. The unique pharmacological and pharmacokinetic profile of teneligliptin: implications for clinical practice. Drugs. 2019;79(7):733-750. doi:10.1007/s40265-019-01086-0

7. Tanaka K, Okada Y, Mori H, et al. Efficacy of linagliptin and teneligliptin for glycemic control in type 2 diabetic patients with chronic kidney disease: assessment by continuous glucose monitoring; a pilot study. Diabetol Int. 2016;7(4):368-374. doi:10.1007/ s13340-016-0258-y

8. Rosenstock J, Perkovic V, Johansen OE, et al. Effect of linagliptin vs placebo on major cardiovascular events in adults with type 2 diabetes and high cardiovascular and renal risk: the CARMELINA randomized clinical trial. JAMA. 2019;321(1):69-79. doi:10.1001/ jama.2018.18269

9. von Elm E, Altman DG, Egger M, et al. The Strengthening the Reporting of Observational Studies in Epidemiology (STROBE) statement: guidelines for reporting observational studies. PLoS Med. 2007;4(10):e296. doi:10.1371/journal.pmed.0040296

10. Matsuo S, Imai E, Horio M, et al. Revised equations for estimated GFR from serum creatinine in Japan. Am J Kidney Dis. 2009;53 (6):982-992. doi:10.1053/j.ajkd.2008.12.034

11. Kadowaki T, Kondo K. Efficacy, safety and dose-response relationship of teneligliptin, a dipeptidyl peptidase-4 inhibitor, in Japanese patients with type 2 diabetes mellitus. Diabetes Obes Metab. 2013;15 (9):810-818. doi:10.1111/dom.12092

12. Groop PH, Cooper ME, Perkovic V, Emser A, Woerle HJ, von Eynatten M. Linagliptin lowers albuminuria on top of recommended standard treatment in patients with type 2 diabetes and renal dysfunction. Diabetes Care. 2013;36(11):3460-3468. doi:10.2337/ dc13-0323

\section{Publish your work in this journal}

Pragmatic and Observational Research is an international, peerreviewed, open access journal that publishes data from studies designed to reflect more closely medical interventions in realworld clinical practice compared with classical randomized controlled trials (RCTs). The manuscript management system is completely online and includes a very quick and fair peer-review system. Visit $\mathrm{http}: / /$ www.dovepress.com/testimonials.php to read real quotes from published authors. 